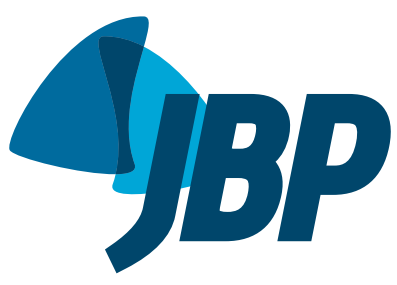

1. Departamento de Pneumologia, Hospital de Santa Maria, Centro Hospitalar Lisboa Norte, Lisboa, Portugal.

Submitted: 9 March 2016. Accepted: 15 August 2016

Study carried out in the Departamento de Pneumologia, Hospital de Santa Maria, Centro Hospitalar Lisboa Norte, Lisboa, Portugal.

\section{Spontaneous pneumomediastinum: experience in 18 patients during the last 12 years}

Patrícia Dionísio', Luís Martins', Susana Moreira', Alda Manique ${ }^{1}$ Rita Macedo ${ }^{1}$, Fátima Caeiro ${ }^{1}$, Luísa Boal' ${ }^{1}$, Cristina Bárbara ${ }^{1}$

\begin{abstract}
Objective: To characterize clinically all of the patients with spontaneous pneumomediastinum (SPM) admitted to an adult pulmonology ward in Lisbon, Portugal. Methods: This was a retrospective descriptive study of all adult patients ( $\geq 18$ years of age) diagnosed with SPM between January of 2004 and September of 2015. Results: At least one predisposing factor was identified in most (88.9\%) of the 18 patients who presented with SPM during the study period. With regard to precipitating factors, bouts of cough were present in $50.0 \%$ of the patients. Other precipitating factors included a sudden increase in tobacco consumption, inhaled drug use, occupational inhalation of varnish fumes, intense exercise, and vomiting. The most common complaints were dyspnea (in 83.3\%) and chest pain (in 77.8\%). Other complaints included cough, neck pain, dysphagia, and odynophagia. Subcutaneous emphysema was found in most of the patients. The diagnosis of SPM was based on chest X-ray findings in $61.1 \%$ of the patients. Conclusions: Although SPM is a rare condition, it should be considered in the differential diagnosis of chest pain and dyspnea. It can develop without a triggering event or conclusive findings on a chest X-ray, which is usually sufficient for diagnosis.
\end{abstract}

Keywords: Mediastinal emphysema; Subcutaneous emphysema; Dyspnea.

\section{INTRODUCTION}

Spontaneous pneumomediastinum (SPM) or spontaneous mediastinal emphysema is a rare condition characterized by free air in the mediastinum not preceded by thoracic trauma, surgery, or any other medical procedure. ${ }^{(1-3)}$ It was first described by Louis Hamman in 1939 , which is why it is also known as Hamman's syndrome..$^{(1,2,4-7)}$ However, secondary pneumomediastinum had previously been reported as a traumatic complication, by René Laennec in $1819 .{ }^{(7)}$ SPM is a benign and usually self-limiting condition that primarily affects young males. In many cases, the precipitating factor or underlying disease cannot be identified. ${ }^{(1,8)}$ The literature describes pre-existing factors/ conditions that facilitate the onset of SPM, known as predisposing factors, and events/conditions that trigger it, known as precipitating factors. ${ }^{(7)}$

The pathophysiology of SPM was first described in 1944 by Macklin and Macklin, who suggested the presence of an alveolar-interstitial pressure gradient. Increased airway pressure leads to alveolar rupture and, consequently, dissection of air along the bronchovascular sheath toward the mediastinum, which can extend to the cervical subcutaneous tissue, pleura, pericardium, peritoneal cavity, and epidural space. $(2,5,9,10)$ The increase in pressure in the intrapleural space and in the airway is due to predisposing factors, such as smoking, bronchial asthma, respiratory infection, and interstitial lung disease, ${ }^{(1,2)}$ in combination with precipitating factors, such as bouts of cough, emesis, and vigorous exercise. ${ }^{(4)}$ In some cases, no identifiable cause is found. (4) SPM has also been associated with inhaled drug use-including marijuana, cocaine, and ecstasy use-which is related to various mechanisms, including performance of the Valsalva maneuver, strong pulmonary vasoconstriction, and direct effects on the alveolar membrane. ${ }^{(6)}$ Although SPM has been associated with noninvasive ventilation ${ }^{(11)}$ and dental procedures involving the use of a high-speed air-turbine handpiece, ${ }^{(12,13)}$ such cases are best classified as iatrogenic.

The most common symptoms and signs of SPM are dyspnea, chest pain, neck pain, and subcutaneous emphysema. ${ }^{(4,5,14)}$ On chest auscultation, a crunching sound synchronous with the heartbeat (Hamman's sign) can sometimes be heard. ${ }^{(1,4)}$ Pneumomediastinum is typically diagnosed on the basis of posteroanterior and lateral chest $\mathrm{X}$-rays. ${ }^{(3)}$

SPM can go unnoticed for various reasons. First, it is a rare condition, the incidence of which ranges from $1: 7,000$ to $1: 45,000$ hospital admissions. ${ }^{(7)}$ Second, it is a poorly recognized disease; the only published studies to date are reports of isolated clinical cases or small case series. Finally, patient complaints are not specific to SPM and are common in numerous other cardiopulmonary diseases. ${ }^{(3,5,8,14)}$

The prognosis is usually excellent with conservative treatment, i.e., rest, oxygen therapy, and analgesia (if

Correspondence to:

Patrícia Alexandra Vieira Dionísio. Departamento de Pneumologia, Hospital de Santa Maria, Centro Hospitalar Lisboa Norte, Avenida Prof. Egas Moniz, 1649-035, Lisboa Portugal.

Tel.: 351 96029-2138. E-mail: patriciadionisio1@gmail.com

Financial support: None. 
necessary). Although SPM is rare, patients should be monitored for complications such as hypertensive pneumothorax, pneumopericardium, pneumomediastinum, pneumoperitoneum, pneumorrhachis, and mediastinitis. Although the risk of recurrence is low, secondary causes should be excluded, ${ }^{(2,4)}$ including iatrogenic/traumatic perforation of the esophagus or trachea/respiratory tract and intrathoracic infection. ${ }^{(2)}$

The objective of the present study was to characterize clinically all SPM patients admitted to the Pulmonology Ward of Centro Hospitalar Lisboa Norte Hospital de Santa Maria, in the city of Lisbon, Portugal, in the last 11 years, by analyzing their progression and response to conservative treatment.

\section{METHODS}

This was a retrospective descriptive study aimed at identifying all of the adult patients ( $\geq 18$ years of age) who were admitted to the aforementioned ward with a diagnosis of SPM in the period between January of 2004 and September of 2015. To that end, all discharge documents issued during the study period were reviewed. Secondary causes of pneumomediastinum were excluded, including thoracic trauma, surgery, invasive tests, and upper aerodigestive tract manipulation. All patients were admitted via the emergency department of our hospital, either directly or referred by other hospitals.

All data were collected in accordance with a previously established protocol and included the following information: demographic data; possible predisposing and precipitating factors; symptoms and signs; additional diagnostic tests; treatment received during hospital stay; clinical course; length of hospital stay; and readmissions. When data on symptoms and signs were collected, information regarding the presence of cough, dyspnea, dysphagia, odynophagia, neck pain, chest pain, and subcutaneous emphysema was actively sought. Outpatient files were also reviewed, in order to identify any recurrences.

Statistical analysis was performed with Microsoft Office Excel 2013, proportions, means, and standard deviations being calculated. The study was approved by the Research Ethics Committee of Centro Hospitalar Lisboa Norte.

\section{RESULTS}

Over a period of 11 years and 9 months, a total of $1,835,817$ adult patients ( $\geq 18$ years of age) sought emergency room treatment at Hospital de Santa Maria, patients presenting with gynecological/obstetric conditions not being taken into account. During the same period, a total of 8,581 patients were admitted to our pulmonology ward. Of those, 18 were diagnosed with SPM. Therefore, the incidence of SPM in our study was approximately $1: 102,000$. Of the 18 patients diagnosed with SPM, $66.7 \%$ were male (the youngest being 18 years old and the oldest being 87 years old), and the mean age was $35.4 \pm 24.7$ years. The mean length of hospital stay was $10.5 \pm 9.9$ days.

As can be seen in Table 1, at least one predisposing factor was identified in most of the patients (88.9\%), as follows: $44.4 \%$ were current smokers; $22.2 \%$ were former smokers; $44.4 \%$ had a history of recent respiratory infection; $27.8 \%$ had a diagnosis of bronchial asthma; $22.2 \%$ had a history of bronchial hyperreactivity (without bronchial asthma); and 11.1\% had a history of interstitial lung disease. With regard to precipitating factors (Table 2 ), $50.0 \%$ of the cases of SPM were related to bouts of cough; 2 were related to bouts of vomiting; 2 were related to inhaled drug use; 1 was related to a sudden increase in tobacco consumption; 1 was related to occupational inhalation of varnish fumes; and 1 was related to intense physical activity. In $2(11.1 \%)$ of the patients, no potential precipitating factor was identified.

As can be seen in Table 3, the following symptoms were identified: dyspnea, in $83.3 \%$ of the patients; chest pain, in $77.8 \%$; cough, in $55.6 \%$; neck pain, in $55.6 \%$; dysphagia, in $27.8 \%$; and odynophagia, in $16.7 \%$. Physical examination revealed subcutaneous emphysema in $83.3 \%$ and Hamman's sign in only 1 patient (Table 3).

All patients underwent the following tests: complete blood count; blood coagulation testing; renal function testing; hepatic function testing; determination of serum electrolyte levels; and arterial blood gas analysis. At emergency room admission, mean leukocyte count was $12,540 \times 10^{9}$ cells/L (range, 3,500 $\times 10^{9}$ cells/L to $20,570 \times 10^{9}$ cells/L). Of the total of patients, 10 had leukocytosis and 12 had neutrophilia (> 70\% of the relative leukocyte count). All patients underwent chest X-rays, diagnosis being based on chest X-ray findings in $11(61.1 \%)$. The remaining patients underwent chest CT scans in order to clarify the diagnosis. Of the 2 patients in whom SPM was related to a bout of vomiting, 1 underwent barium esophagography and 1 underwent upper gastrointestinal endoscopy, which excluded esophageal discontinuity. In most (11) of the patients, there were no complications directly related to SPM. Of the remaining 7 patients, 5 had pneumothorax and 2 had pneumorrhachis. ${ }^{(15)}$ Although 1 patient died-an 81-year-old male with a history of chronic hypersensitivity pneumonitis-the cause of death was unrelated to SPM. His death was attributed to nosocomial pneumonia with type II respiratory failure. Although the patient required noninvasive ventilation, SPM had been diagnosed before the initiation of noninvasive ventilation.

All of the patients received conservative medical treatment for SPM. Treatment consisted of bed rest, analgesia, oxygen therapy, and serial chest X-rays. Of the 18 patients, 7 received antibiotic therapy for concomitant respiratory tract infection. There were no recurrences in the 13 patients who were subsequently followed as outpatients, the duration of follow-up having ranged from 1 month to 76 months. Of the 
Table 1. Predisposing factors for spontaneous pneumomediastinum $(\mathrm{N}=18)$.

\begin{tabular}{lll}
\multicolumn{1}{c}{ Predisposing factor } & $\mathbf{n}$ & $\%$ \\
Current smoking & 8 & 44.4 \\
Recent respiratory infection & 8 & 44.4 \\
Bronchial asthma & 5 & 27.8 \\
Past smoking & 4 & 22.2 \\
Bronchial hyperreactivity (without bronchial asthma) & 4 & 22.2 \\
Interstitial lung disease & 2 & 11.1 \\
\hline
\end{tabular}

Table 2. Precipitating factors for spontaneous pneumomediastinum $(\mathrm{N}=18)$.

\begin{tabular}{|c|c|c|}
\hline Precipitating factor & $\mathbf{n}$ & $\%$ \\
\hline Cough & 9 & 50.0 \\
\hline Vomiting & 2 & 11.1 \\
\hline Inhaled drug use & 2 & 11.1 \\
\hline Intense exercise & 1 & 5.6 \\
\hline Occupational inhalation of varnish fumes & 1 & 5.6 \\
\hline Sudden increase in tobacco consumption & 1 & 5.6 \\
\hline Unidentified & 2 & 11.1 \\
\hline
\end{tabular}

Table 3. Symptoms and signs present on admission $(\mathrm{N}=18)$.

\begin{tabular}{|c|c|c|}
\hline Symptoms and signs & $\mathbf{n}$ & $\%$ \\
\hline Dyspnea & 15 & 83.3 \\
\hline Subcutaneous emphysema & 15 & 83.3 \\
\hline Chest pain & 14 & 77.8 \\
\hline Cough & 10 & 55.6 \\
\hline Neck pain & 10 & 55.6 \\
\hline Dysphagia & 5 & 27.8 \\
\hline Odynophagia & 3 & 16.7 \\
\hline Hamman's sign & 1 & 5.6 \\
\hline
\end{tabular}

remaining 5 patients, 4 were lost to follow-up and 1 died (as previously mentioned).

As previously mentioned, there have been few reports of pneumomediastinum related to dental procedures involving the use of high-speed air-turbine handpieces. Therefore, we decided to mention here the case of a patient who was admitted to our pulmonology ward during the study period. It was not included in our statistical analysis because it was considered to be a case of iatrogenic/secondary pneumomediastinum. The patient was a 36-year-old nonsmoking female who had had sudden-onset cervical subcutaneous emphysema, chest pain, and neck pain during a dental cleaning procedure with the use of a high-speed air-turbine handpiece. Diagnosis was based on chest $\mathrm{X}$-ray findings, and the patient received conservative treatment. She had no recurrence during her hospital stay and was discharged after 5 days.

\section{DISCUSSION}

Although the true incidence of SPM is unknown, it is probably underestimated because few practitioners are aware of this condition, the diagnosis of which requires a high level of suspicion. ${ }^{(16)}$ In Portugal, no published studies have been found that have examined this issue, and there had been no estimates of the incidence of SPM in the country before the present study. The incidence of SPM in our study $(1: 102,000)$ is much lower than that reported in other studies. Although this suggests that SPM is underdiagnosed, further studies are needed in order to confirm that.

The demographic characteristics of our sample are also different from those reported in the literature. The mean age was relatively higher in the present study, which is probably due to the fact that our patients varied widely in age. In agreement with other studies, ${ }^{(3,17)}$ there was a predominance of males in the present study $(2: 1)$.

In most studies, the proportion of SPM cases associated with a precipitating factor ranges from $21.0 \%$ to $75.0 \%$. $^{(2,4-6,14,17,18)}$ In the present study, however, that proportion was substantially higher (88.9\%). This might be due to the fact that all of the patients in the present study were admitted to a pulmonology ward where precipitating factors were actively investigated. Most of the cases of SPM in the present study were found to be related to the Valsalva maneuver performed during bouts of cough or vomiting, a finding that is in accordance with other studies..$^{(5,6,10,14,19)}$ In the cases in which SPM was associated with a sudden increase in tobacco consumption, with occupational inhalation of varnish fumes, or with inhaled drug use, SPM was also attributed to the Valsalva maneuver, performed 
either during inhalation or during the bouts of cough triggered by airway irritation. While reviewing the discharge documents, we found no reference to patients being systematically questioned about inhaled drug abuse and the type(s) of drug(s) used.

In a systematic review of 27 studies of SPM (including a total of 600 patients), at least one predisposing factor was identified in $22.0 \%$ of the cases. The most common predisposing factor was bronchial asthma, followed by interstitial lung disease, COPD, bronchiectasis, bullae, thoracic neoplasms, cystic disease, and respiratory tract infection. (17) In the present study, the most common predisposing factor was current or past smoking (in 12 of the 18 patients), which led to respiratory tract inflammation and, consequently, bouts of cough. The fact that current or past smoking was considered a predisposing factor for SPM in the present study significantly increased the number of patients with at least one predisposing factor for SPM.

In all of the patients in the present study, SPM presented as an acute or subacute condition with no signs of hemodynamic instability or exuberant inflammatory response. With regard to the symptoms most commonly associated with SPM, our findings are consistent with those of most studies, $(2-6,10,14,17-20)$ as is our finding of a mild to moderate inflammatory response to SPM. ${ }^{(4-6,18)}$

In the present study, the diagnosis of SPM was based on chest X-ray findings in most (61.1\%) of the patients. Thoracic CT was used only in cases of uncertainty, as recommended elsewhere. ${ }^{(6,16)}$ However, one group of authors ${ }^{(9)}$ reported that pneumomediastinum was visible on chest $X$-rays in only $52.9 \%$ of patients. This difference in proportions across studies might be due to the different medical or surgical specialties of the physicians involved and to the different hospital departments to which patients were admitted.

In the present study, the clinical course of SPM was invariably benign, and all patients received conservative treatment. The mean length of hospital stay was 10.5 \pm 9.9 days, significantly longer than that reported in a systematic review published in 2013 (4.1 \pm 2.3 days). ${ }^{(17)}$ This discrepancy is explained by the fact that the mean age of the patients in the present study was higher and by the presence of associated conditions, such as interstitial lung diseases. In the
13 patients who were followed as outpatients, there were no recurrences, a finding that is consistent with the literature. ${ }^{(17)}$

Invasive procedures have rarely been described in SPM. Their use is reserved for tension pneumomediastinum with respiratory distress, ${ }^{(21)}$ significant cardiorespiratory compromise, such as in cases of pneumopericardium resulting in air tamponade, (22) and specific cases of esophageal tear. ${ }^{(23)}$

Although SPM is a rare condition, it should be considered in the differential diagnosis of chest pain and dyspnea, which focuses on cardiovascular and pulmonary sources, including acute coronary syndromes, pericarditis, aortic dissection, pulmonary thromboembolism, pneumonia, pleural effusion, and pneumothorax. ${ }^{(2)}$ Musculoskeletal pain, gastroesophageal reflux disease, esophageal perforation, and spasm are also included in the differential diagnosis. ${ }^{(2)}$

The present study confirms that SPM can develop without a triggering event and with no conclusive findings on a chest X-ray. Because they are usually sufficient for diagnosis, posteroanterior and lateral chest $X$-rays should be performed first, thoracic CT being reserved for cases in which chest $X$-ray findings are inconclusive. Likewise, esophagography and thoracic CT should be performed only if there is a history or clinical evidence of esophageal rupture, whereas fiberoptic bronchoscopy and thoracic CT should be performed only in cases of suspected tracheal rupture. Despite an excellent prognosis with conservative treatment and a low risk of recurrence, secondary causes should be excluded, and patients should be monitored for complications. Our findings suggest that it might be useful for pulmonology departments to implement protocols that allow early diagnosis of pneumomediastinum by including specific questions regarding inhaled drug use, as well as drug testing.

\section{ACKNOWLEDGMENTS}

The authors would like to thank the staff of the Emergency and Pulmonology Departments of Hospital de Santa Maria for providing all of the information needed in order to conduct this retrospective study. The authors gratefully acknowledge the support and assistance of Dr. Isabel Correia in the execution of this study.

\section{REFERENCES}

1. Fishman AP, Elias JA, Fishman JA, Grippi MA, Senior RM, Pack Al. Fishman's Pulmonary Disease and Disorders. 4th ed. Philadelphia: McGraw-Hill Medical; 2008

2. Caceres M, Ali SZ, Braud R, Weiman D, Garrett HE Jr. Spontaneous pneumomediastinum: a comparative study and review of the literature. Ann Thorac Surg. 2008;86(3):962-6. http://dx.doi. org/10.1016/j.athoracsur.2008.04.067

3. Sahni S, Verma S, Grullon J, Esquire A, Patel P, Talwar A. Spontaneous pneumomediastinum: time for consensus. N Am J Med Sci. 2013;5(8):460-4. http://dx.doi.org/10.4103/1947-2714.117296

4. Ryoo JY. Clinical analysis of spontaneous pneumomediastinum Tuberc Respir Dis (Seoul). 2012;73(3):169-73. http://dx.doi.

org/10.4046/trd.2012.73.3.169

5. Macia I, Moya J, Ramos R, Morera R, Escobar I, Saumench $J$, et al. Spontaneous pneumomediastinum: 41 cases. Eur $J$ Cardiothorac Surg. 2007;31(6):1110-4. http://dx.doi.org/10.1016/j. ejcts.2007.03.008

6. Bakhos CT, Pupovac SS, Ata A, Fantauzzi JP, Fabian T. Spontaneous pneumomediastinum: an extensive workup is not required. J Am Coll Surg. 2014;219(4):713-7. http://dx.doi.org/10.1016/j. jamcollsurg.2014.06.001

7. Meireles J, Neves S, Castro A, França M. Spontaneous pneumomediastinum revisited. Respir Med CME. 2011;4(4):181-3. http://dx.doi.org/10.1016/j.rmedc.2011.03.005 
8. Koullias GJ, Korkolis DP, Wang XJ, Hammond GL. Current assessment and management of spontaneous pneumomediastinum: experience in 24 adult patients. Eur J Cardiothoracic Surg. 2004;25(5):852-5. http://dx.doi.org/10.1016/j.ejcts.2004.01.042

9. Al-Mufarrej F, Badar J, Gharagozloo F, Tempesta B, Strother E, Margolis M. Spontaneous pneumomediastinum: diagnostic and therapeutic interventions. J Cardiothorac Surg. 2008;3:59. http:// dx.doi.org/10.1186/1749-8090-3-59

10. Kelly S, Hughes S, Nixon S, Paterson-Brown S. Spontaneous pneumomediastinum (Hamman's syndrome). Surgeon. 2010;8(2):636. http://dx.doi.org/10.1016/j.surge.2009.10.007

11. Ruggeri P, Girbino G. Fatal pneumomediastinum associated with use of noninvasive mechanical ventilation. Respirol Case Rep. 2014;2(4):126-8. http://dx.doi.org/10.1002/rcr2.73

12. Aslaner MA, Kasap GN, Demir C, Akkaş M, Aksu NM. Occurrence of pneumomediastinum due to dental procedures. Am J Emerg Med. 2015;33(1):125.e1-3. http://dx.doi.org/10.1016/j.ajem.2014.05.055

13. Johannesma PC, Vonk Noordegraaf A. Pneumomediastinum and pneumopericardium due to high-speed air turbine drill used during a dental procedure. Ann Thorac Surg. 2014;98(6):2232. http://dx.doi. org/10.1016/j.athoracsur.2014.08.013

14. Iyer VN, Joshi AY, Ryu JH. Spontaneous pneumomediastinum: analysis of 62 consecutive adult patients. Mayo Clin Proc. 2009;84(5):417-21. http://dx.doi.org/10.1016/S0025-6196(11)605600

15. Martins L, Dionísio P, Moreira S, Manique A, Correia I, Bárbara C Case Report An Unusual Association in an Uncommon Disease: Two Cases of Spontaneous Pneumomediastinum Associated with Pneumorrhachis. Case Rep Pulmonol. 2016;2016:5092157.
16. Esayag $Y$, Furer $V$, Izbicki $G$. Spontaneous pneumomediastinum : is a chest X-ray enough ? Isr Med Assoc J. 2008;10(8-9):575-8.

17. Dajer-Fadel WL, Argüero-Sánchez R, Ibarra-Pérez C, Navarro-Reynoso FP. Systematic review of spontaneous pneumomediastinum: a survey of 22 years' data. Asian Cardiovasc Thorac Ann. 2014;22(8):997-1002. http://dx.doi.org/10.1177/0218492313504091

18. Takada K, Matsumoto S, Hiramatsu T, Kojima E, Watanabe H, Sizu $\mathrm{M}$, et al. Management of spontaneous pneumomediastinum based on clinical experience of 25 cases. Respir Med. 2008;102(9):1329-34. http://dx.doi.org/10.1016/j.rmed.2008.03.023

19. Lee SY, Sheu CY, Wu CL, Hung $C L$, Weng $Y L$, Lin $C C$, et al. Spontaneous pneumomediastinum: a clinical radiologic analysis Int J Gerontol. 2008;2(4):222-8. http://dx.doi.org/10.1016/S18739598(09)70011-0

20. Banki F, Estrera AL, Harrison RG, Miller CC 3rd, Leake SS, Mitchell $K G$, et al. Pneumomediastinum: etiology and a guide to diagnosis and treatment. Am J Surg. 2013;206(6):1001-6; discussion 1006. http:// dx.doi.org/10.1016/j.amjsurg.2013.08.009

21. Dondelinger RF, Coulon M, Kurdziel JC, Hemmer M. Tension mediastinal emphysema: emergency percutaneous drainage with CT guidance. Eur J Radiol. 1992;15(1):7-10. http://dx.doi. org/10.1016/0720-048X(92)90193-D

22. Cummings RG, Wesly RL, Adams DH, Lowe JE. Pneumopericardium resulting in cardiac tamponade. Ann Thorac Surg. 1984;37(6):511-8 http://dx.doi.org/10.1016/S0003-4975(10)61146-0

23. Aga Z, Avelino J, Darling GE, Leung JJ. An Unusual Case of Spontaneous Esophageal Rupture after Swallowing a Boneless Chicken Nugget. Case Rep Emerg Med. 2016;2016:5971656. http:// dx.doi.org/10.1155/2016/5971656 